\title{
Neonatal head ultrasound: systematic approach to congenital Central Nervous System anomalies. A pictorial essay.
}

\author{
Hye-Kyung Yoon, Seong Whi Cho
}

Department of Radiology, Kangwon National University Hospital, 200-722, Chuncheon-si, Gangwon-do, South Korea

\begin{abstract}
Brain ultrasound is widely used for the screening of prematurely born babies. Although the best imaging modality for the central nervous system anomaly is brain MRI, the first imaging study in the post-natal period is brain ultrasonography in most cases. Anomalies could be found incidentally on screening ultrasound, or in those cases already suspected on prenatal ultrasound. In order not to miss congenital structural abnormalities of the brain on screening ultrasound, systematic approaches would be very helpful. The ventricles and sylvian fissures are very important structures to suspect central nervous system anomalies: they are symmetric structures so we should look for any asymmetry or maldevelopment. And then, on sagittal images, the midline structures including the corpus callosum and cerebellar vermis should be observed carefully. Finally, we should look for any abnormality in gyration or cortical development. Skull defect with herniation of intracranial contents, a spectrum of encephalomeningocele, could be alsoidentified on ultrasound. Congenital infections such as cytomegalovirus infectionmay showventriculomegaly and malformation of the cortical development on imaging studies.
\end{abstract}

Keywords: ultrasonography, brain, congenital abnormalities

\section{Introduction}

Brain ultrasound is a very useful initial screening modality for evaluating the neonatal brain, especially in premature infants who are under increased risk of intracranial hemorrhage [1]. Brain magnetic resonance imaging (MRI) is the best modality of choice for the diagnosis of congenital structural abnormalities. With the aid of highend ultrasound scanner, the brain structures including the cortices have become more clearly seen on ultrasound, so it can make us identify more subtle changes to suspect congenital structural abnormalities. Routine coronal and sagittal images are used for detecting congenital structural abnormalities; sometimes, a scan through the mastoid fontanelle is required to better see the cerebellar lesions and the posterior fossa malformations [2]. Stepwise ap-

Received 14.11.2015 Accepted 25.12.2015

Med Ultrason

2016, Vol. 18, No 3, 386-393

Corresponding author: Seong Whi Cho, M.D.

Department of Radiology

Kangwon National University Hospital

Baengnyeong-ro 156, Chuncheon-si,

Gangwon-do, 200-722, South Korea

E-mail: chosw@kangwon.ac.kr proaches from the ventricles and sylvian fissures to the midline structures and cortical gyri would be very helpful for the personnel who perform head ultrasound daily to suspect any congenital structural abnormalities of the neonatal brain.

\section{Ventricles}

\section{Normal ventricles}

The lateral ventricles in most infants measure less than 2-3mm in diameter and appear slit-like or crescentic (fig 1). Mild ventricular asymmetry is considered to be a normal variantwhich has been described in up to $90 \%$ of normal newborn infants $[1,3,4]$. It has been reported that the larger ventricle is more commonly on the left side [5].

The frontal horns and body portions of the lateral ventricles are divided by the midline septum pellucidum. Cavum septum pellucidum and vergae begin to close at 6 months of gestation and almost 90 percent of them fuse by 2 to 6 months of postnatal period although they can persist into adult life. Practically, it is commonly encountered when performing head ultrasound in newborn period.

In many cases of congenital structural abnormalities, the ventricles are abnormal in shape and/or size with- 

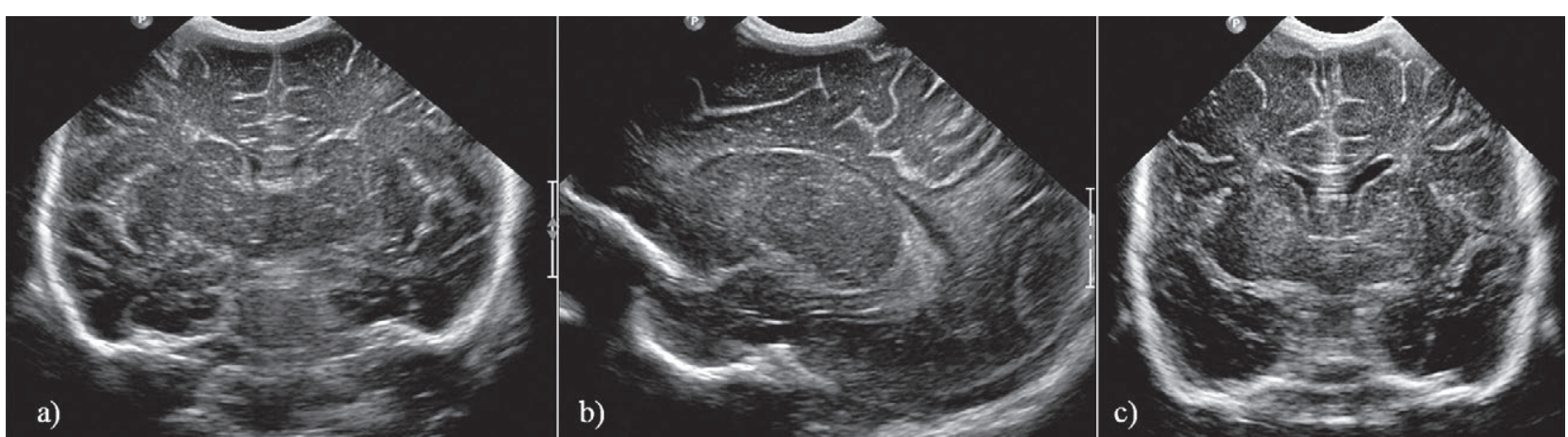

Fig 1. Normal ventricles. Coronal (a) and sagittal (b) imagesshow symmetric crescentic lateral ventricles. Mild ventricular asymmetry is considered normal variation (c).

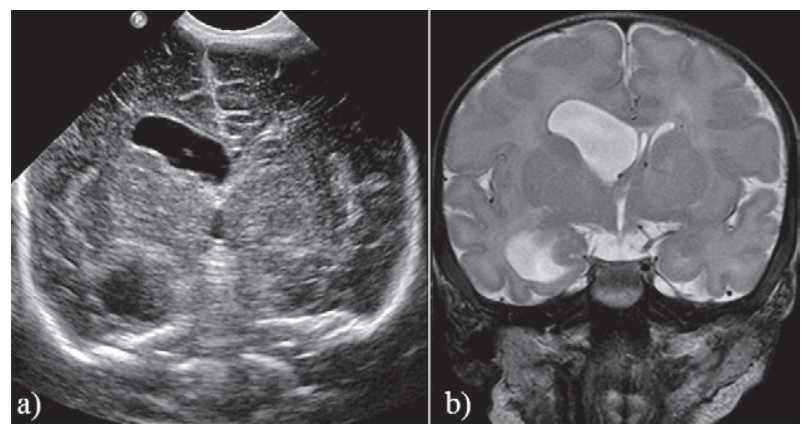

Fig 2. Unilateral agenesis of the foramen of Monro. Coronal ultrasonography (a) and T2-weighted coronal MR image (b) show asymmetric dilatation of the right lateral ventricle with displacement of the septum pellucidum. Also seen is the normal non-dilated third ventricle.

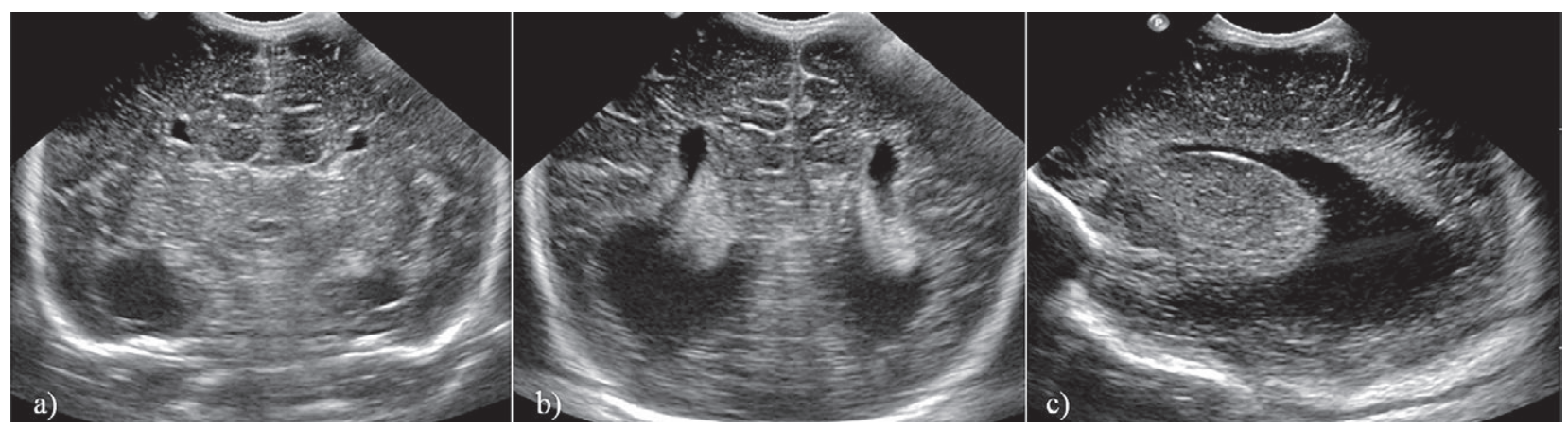

Fig 3. Callosal agenesis with colpocephaly. Coronal ultrasonography through the frontal horns of the lateral ventricles (a) shows widely separated comma-shaped frontal horns. On more posterior coronal scan (b), the trigones of both lateral ventricles are oriented parallel. There is colpocephalic dilatation of the posterior horns which is better seen on the sagittal scan (c).

symmetric dilatation (e.g. colpocephaly in callosal agenesis) or asymmetric distension (e.g. atresia of foramen Monro).

\section{Congenital atresia of foramen Monro}

Unilateral or bilateral occlusion of the foramen of Monro results in dilatation of the lateral ventricles. Other than congenital atresia, infections, vascular malformations, or neoplastic processes can also cause stenosis of the foramen of Monro [6]. In cases of unilateral atresia of the foramen of Monro, there is lateral ventricle dila- tation on the affected side with normal third and fourth ventricles (fig 2).

\section{Callosal agenesis}

The corpus callosum may be absent completely or partially: it may be either isolated or in association with other midline abnormalities. On sonography, the corpus callosum is absent with widely separated lateral ventricles and dilated posterior horns (colpocephaly) (fig 3). The bundles of Probst indent lateral ventricles medially, resultant comma-shaped frontal horns. 


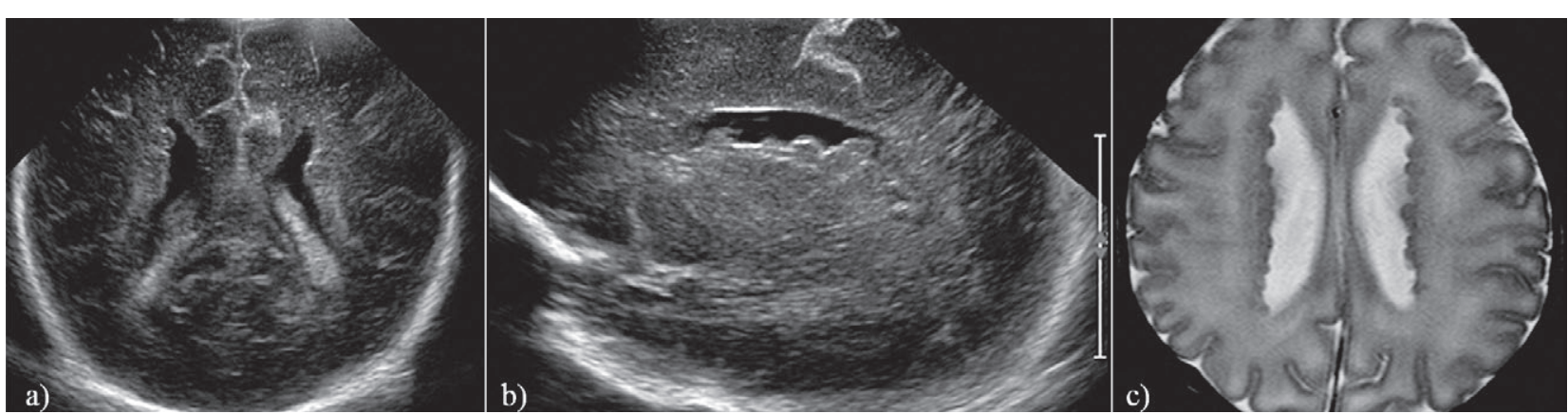

Fig 4. Subependymal (periventricular) heterotopias. Coronal (a) and sagittal (b) ultrasound images show undulating lateral ventricle margins with isoechoic nodular lesions.MR T2-weighted axial image (c )confirms periventricular nodular lesions of cortical signal intensities in keeping with heterotopic gray matter.

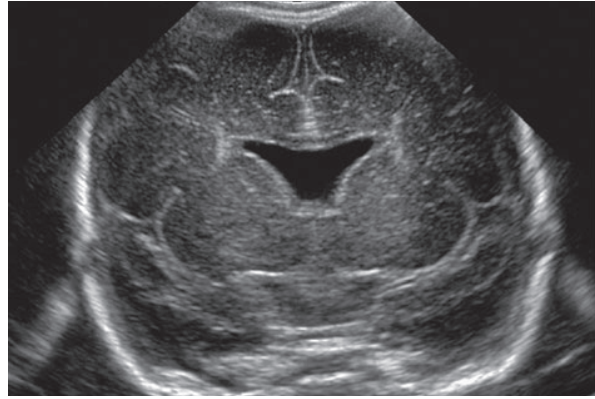

Fig 5. Agenesis of the septum pellucidum. The septum pellucidum is absent on the coronal ultrasound image with flattening of the roof of the lateral ventricle.

\section{Subependymal heterotopia}

Subependymal (periventricular) nodular heterotopias are collections of nerve cells in theperiventricular regions due to the arrest of neural migration [7]. It can be either sporadic or familial. The classic form of bilateral periventricular heterotopia is most common and due to X-linked gene mutations. On ultrasound, the ventricular margins appear undulating with protruding nodules of isoechogenicity to the brain parenchyma (fig 4). The lateral ventricles may appear asymmetric and irregularly dilated.

\section{Agenesis of septum pellucidum}

The development of the septum pellucidum is closely related to the formation of the corpus callosum. Absence of the septum pellucidum may be associated with other anomalies including septooptic dysplasia, schizencephaly, callosal agenesis, etc [8]. Sonographic finding includes non-visualization of the midline septum pellucidum with a box-shaped roof of the lateral ventricles, which is best appreciated on coronal scan (fig 5).

\section{Sylvian fissures}

\section{Normal Sylvian fissures}

The Sylvian fissures are bordered medially by the insula, superiorly by the frontal operculum, and inferi- orly by the temporal lobe. On ultrasound, normal sylvian fissures are seen as symmetric and echogenic Y-shaped structures bilaterally on the coronal image (fig 6). Sylvian fissures in immature babies before 30 gestational weeks are wide and primitive with lack of gyration and sulcation.

\section{Perisylvian polymicrogyria}

Polymicrogyria consists of abnormal disarrayed cortices and is known to be one of the most common malformations of cortical development [9]. Perisylvian cortices are most commonly involved usually in a bilateral symmetric manner. Typical imaging findings on MRI include thickened or over folded cortex, irregular cortical surface, and stippling at the gray-white matter junction. As a result, the adjacent ventricles and sylvian fissures appear dysmorphic in association with the widening of the adjacent extraaxial fluid space. These findings can be identified on head ultrasound as well (fig 7, fig 8).

\section{Midline structures}

\section{Normal midline structures}

Midline sagittal ultrasound reveals the corpus callosum, the third ventricle with the massa intermedia, the brainstem, the fourth ventricle, and the cerebellar vermis (fig 9). The cavum septum pellucidum and cavum vergae can be seen as a fluid-filled structure below the corpus callosum. The cingulated gyrus surrounds the corpus callosum which is a key structure for the diagnosis of callosal agenesis. Echogenic choroid plexus is seen along the roof of the third ventricle. Cisterna magna is located behind the cerebellum, normally measuring $3-8 \mathrm{~mm}$ $[1,10]$.

\section{Agenesis of corpus callosum}

As mentioned before, there can be a complete or partial form of the agenesis of the corpus callosum. On the sagittal image in the complete agenesis, the cingulate gyrus is not present and the gyri appear in a radial fash- 

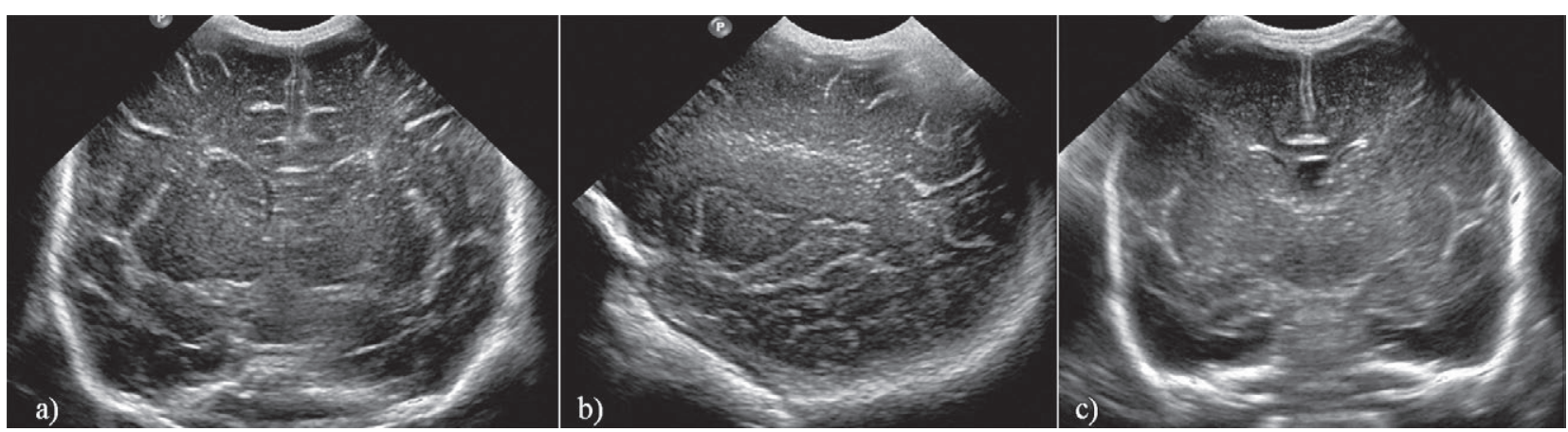

Fig 6. Normal sylvian fissures appear bilateral symmetric Y-shaped echogenic structures on coronal image (a). On parasagittal ultrasonogram (b), the sylvian fissure is marginated by the temporal lobe and the insula. (c) In this 26 week-old prematurely born infant, the sylvian fissures are wide and primitive.



Fig 7. Perisylvian polymicrogyria. Coronal image (a) shows malformed right sylvian fissure with widening of adjacent extraaxial fluid space (arrowheads). MR T2-weighted axial images (b, c) confirmed malformed right sylvian fissure with perisylvian polymicrogyria. Also noted is localized extraaxial fluid space widening adjacent to the malformed gyri with prominent vascular structures.

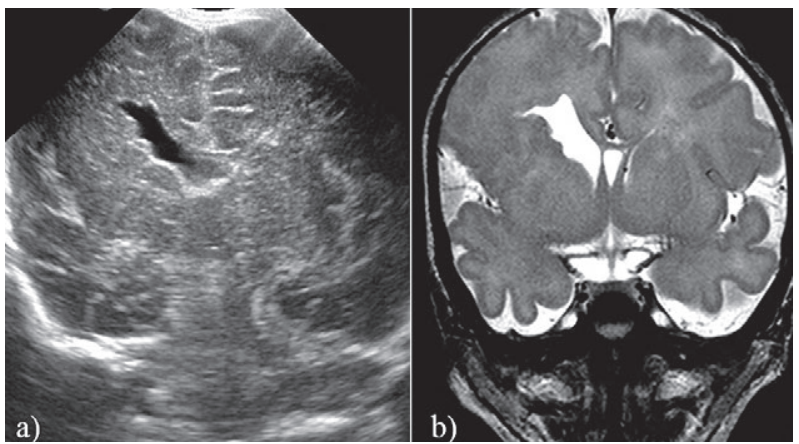

Fig 8. Malformation of cortical development. Coronal sonogram (a) shows asymmetrically dilated irregular right lateral ventricle and malformed right sylvian fissure as compared to the normal opposite side. There is a nodular indentation along the right lateral ventricle margin raising a suspicion of subependymal heterotopias. Corresponding coronal T2-weighted MR image (b) reveals malformed and disorganized tissue on the right side resulting in a dysmorphic right lateral ventricle and right sylvian fissure. Cortical malformations include polymicrogyria and periventricular heterotopias.

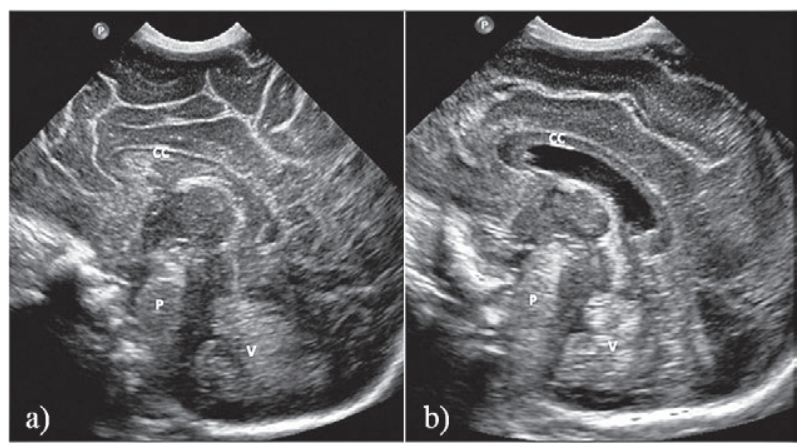

Fig 9. Normal midline sagittal images. Midline sagittal ultrasound images $(\mathrm{a}, \mathrm{b})$ reveal the corpus callosum $(\mathrm{CC})$, third ventricle, pons $(\mathrm{P})$, cerebellar vermis $(\mathrm{V})$, and fourth ventricle. Echogenic choroid plexus is seen along the roof of the third ventricle. Cisterna magna (arrows) is important to exclude a possible Chiari malformation. Cavum septum pellucidum and cavum vergae is seen as a fluid-filled structure below the corpus callosum (b). 


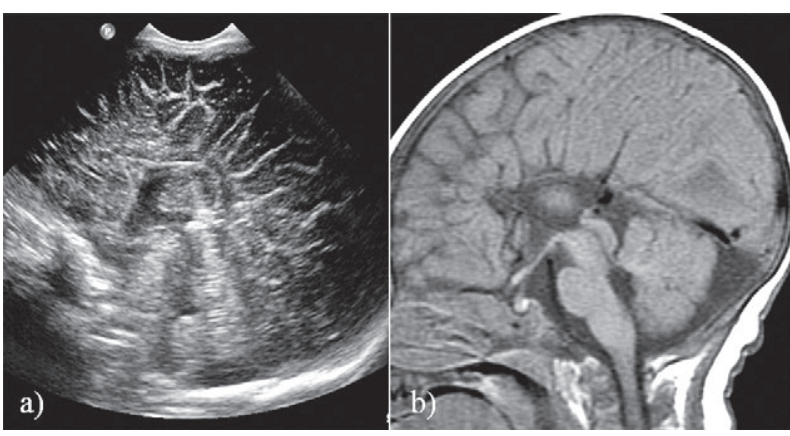

Fig 10. Corpus callosum is absent on midline sagittal ultrasonography (a) and T1-weighted sagittal MR image (b). The cingulate gyrus is not formed with a radial, "spoke-wheel" appearance of the gyri.

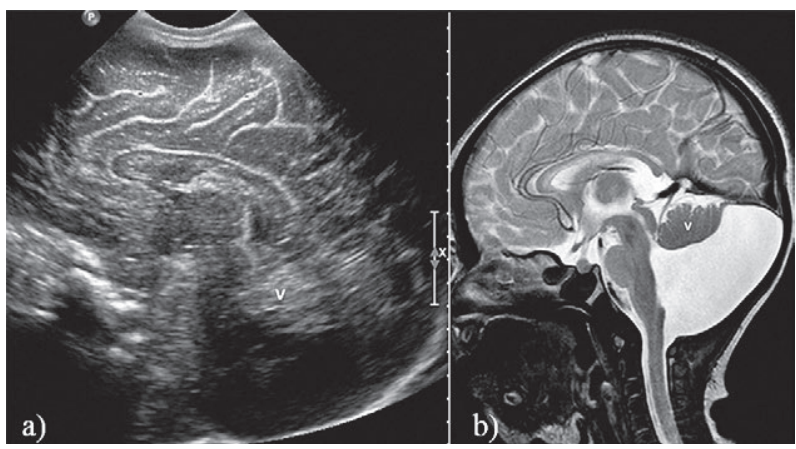

Fig 11. Dandy-Walker malformation. Midline sagittal ultrasound image (a) and corresponding T2-weighted sagittal MR image (b) demonstrate cystic dilatation of the fourth ventricle, inferior vermian agenesis, and increased volume of the posterior fossa. V: cerebellar vermis.

ion (fig 10). The midline cyst or lipoma can be associated with the callosal dysgenesis.

\section{Dandy-Walker complex}

Cystic malformations of the posterior fossa are actually a spectrum of disorders including the DandyWalker malformation, Dandy-Walker variant, mega cistern magna, and retrocerebellar arachnoid cyst [11]. The Dandy-Walker malformation is characterized by the classic triad: complete or partial vermian agenesis; cystic dilatation of the fourth ventricle; and the enlarged posterior fossa (fig 11). In some cases, the cerebellar vermis is dysplastic or almost absent on the mid-sagittal image and the displaced cerebellar tonsil can be misinterpreted as the presence of the cerebellar vermis. Other CNS abnormalities such as the callosal dysgenesis or migration anomaly can be associated in a high percentage of this category. In the Dandy-Walker variant, there is partial agenesis of the cerebellar vermis and the posterior fossa is normal in volume with a less dilated ventricle. On ultrasound, a varying degree of vermian hypoplasia is seen with the free communication of the

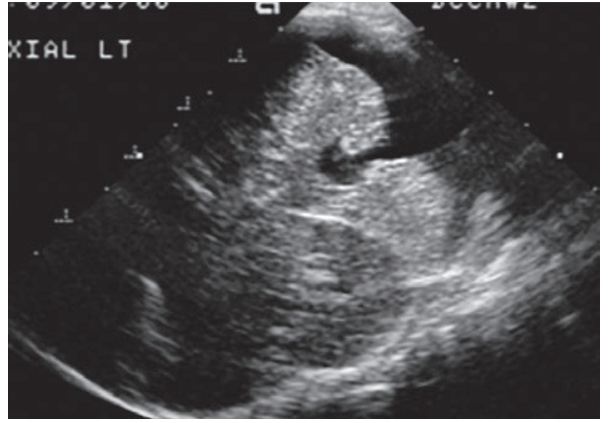

Fig 12. Dandy-Walker variant. Ultrasonography through the mastoid fontanelle shows cystic space in the posterior fossa in communication with the fourth ventricle, so-called a "keyhole" configuration.

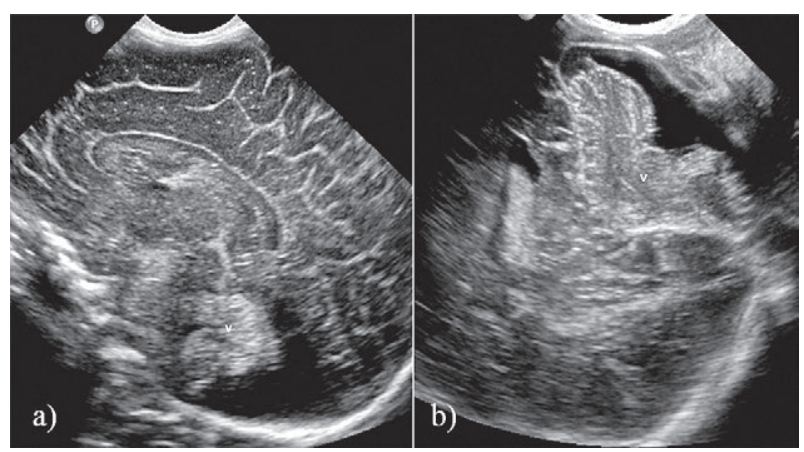

Fig 13. Mega cistern magna. Midline sagittal ultrasonography (a) shows enlarged cystic space behind the cerebellar vermis which is normally formed. Ultrasound through the mastoid fontanelle (b) clearly reveals wide cistern magna around the cerebellum and well-visualized cerebellar vermis (V).

fourth ventricle and retrocerebellar CSF space through prominent valleculae, resulting in a 'key-hole' appearance (fig 12).

Mega cistern magna is a relatively common condition, in about half of all cyst-like posterior fossa malformations. Radiologically, the cistern magna is expanded with morphologically normal cerebellum and vermis (fig 13). Contrary to the mega cistern magna, arachnoid cysts show lack of communication of fluid cavity with the ventricle or subarachnoid space.

\section{Chiari II malformation}

Chiari II malformation is complex involving the hindbrain and spine and is closely related to myelomeningocele [12]. As the normal cerebellum develops within abnormally small posterior fossa, the cerebellum is squeezed out of the posterior fossa. The medulla and cervical cord is stretched inferiorly, resulting in a "cervicomedullary kink". The fourth ventricle is small, compressed with a vertically elongated appearance. The tectum is also distorted with so-called "tectal beaking" (fig 14). Imaging studies including ul- 


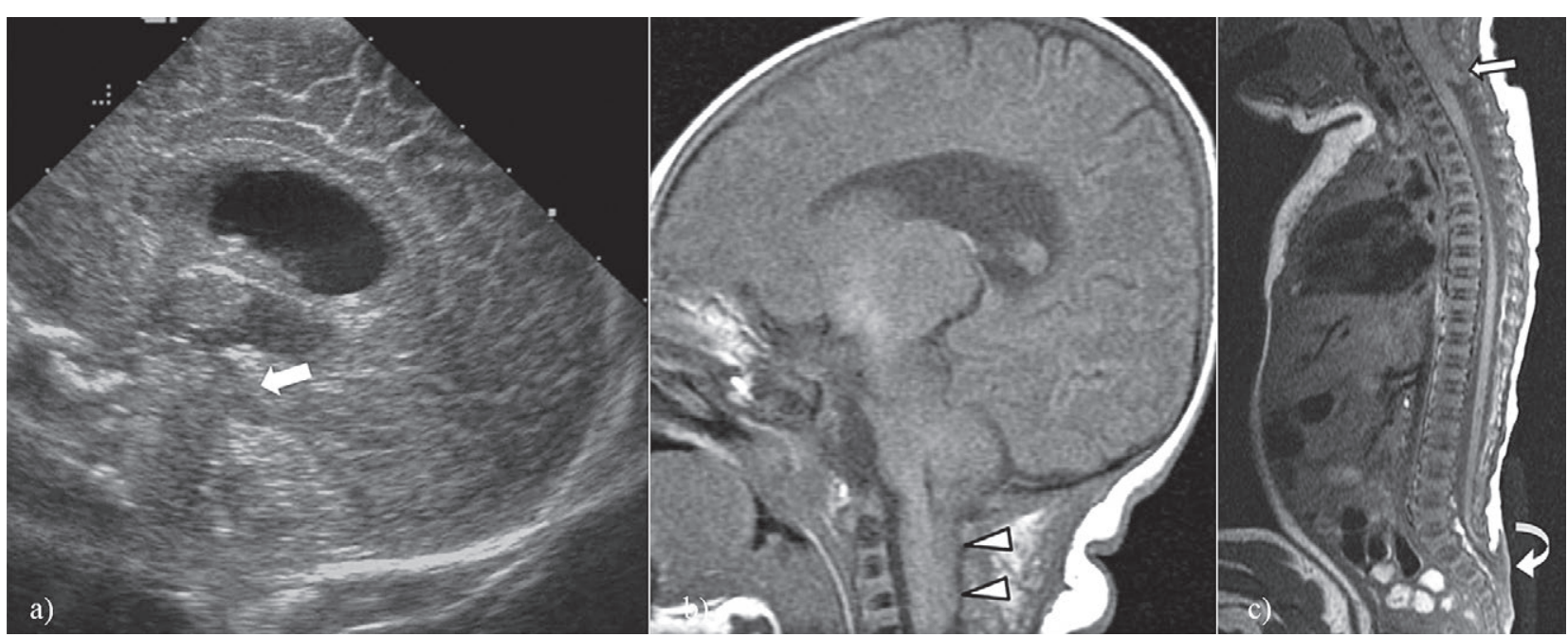

Fig 14. Chiari II malformation. Midline sagittal ultrasound image (a) shows small posterior fossa with obliteration of fluid space in the fourth ventricle and cisterna magna. Tectal bearing is noted (arrow). Sagittal T1-weighted brain (b) and whole spine (c) MR images depict inferiorly herniated cerebellar tissue (arrowheads in b) below the foramen magnum and a cervicomedullary kink (arrow in c). There is a myelomeningocele with a tethered cord at the lumbosacral region (curved arrow in c).

trasound are very important to evaluate Chiari anomaly and hydrocephalus as well as other associated CNS malformations.

\section{Gyrus and cortex}

\section{Normal development of gyrus and cortex}

Gyration takes place in the late pregnancy until term age. In the extremely premature infants with gestational age less than 26 weeks, gyration and sulcation are sparse and the whole brain appears almost agyric and lissencephalic [13]. Maturation process usually occurs in posterior-anterior direction with the occipital region first. As cortical development is on-going, the gyri become more folded and complex in appearance, marginated by thin hyperechogenic sulci. Any event which causes arrest in cortical development results in malformation of the cortical development including lissencephaly, heterotopias, polymicrogyria, schizencephaly, hemimegalencephaly, etc.

\section{Lissencephaly}

Lissencephaly is also known as the agyria-pachygyria complex, representing smooth brain. In agyria, there is a complete absence of the gyri on the cerebral surface whereas a few gyri are presentin the pachygyria (incomplete lissencephaly). Band heterotopia is now considered to be a mild form of lissencephaly [14]. On ultrasound, lissencephaly shows a smooth brain surface with lack of gyration and sulcation, dysmorphic dilated ventricles with or without colpocephalic dilatation, and abnormally wide sylvian fissures (fig 15).

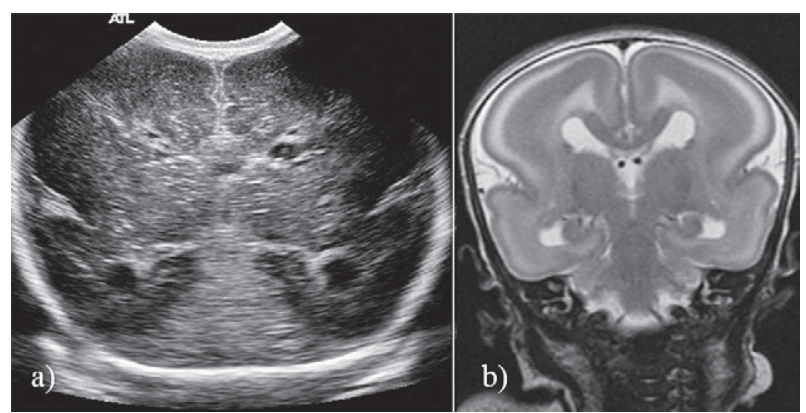

Fig 15. Lissencephaly (agyria-pachygyria). Coronal ultrasound image (a) and corresponding coronal T2-weighted MR image (b) show abnormally shallow and wide sylvian fissures and almost agyric brain surface. Abnormally broad and thick cortices are noted on ultrasound.

\section{Hemimegalencephaly}

Hemimegalencephaly is characterized by the hamartomatous overgrowth of cerebral tissue confined to one cerebral hemisphere as the name implies. It consists of dysplastic tissue with malformations of cortical development in part or total of a cerebral hemisphere. It can be associated with epidermal nevus syndrome, Proteus syndrome, hypomelanosis of Ito, Klippel-Trenaunay-Weber syndrome, and other neurocutaneous syndromes [15]. The lateral ventricle is dilated on the affected side, which is one of differential points from a tumorous condition. Myelination may be accelerated in the affected brain which is best demonstrated on MRI. Ultrasound shows localized or diffuse enlargement of a cerebral hemisphere with dilated ipsilateral ventricles and abnormal broad gyri (fig 16). 


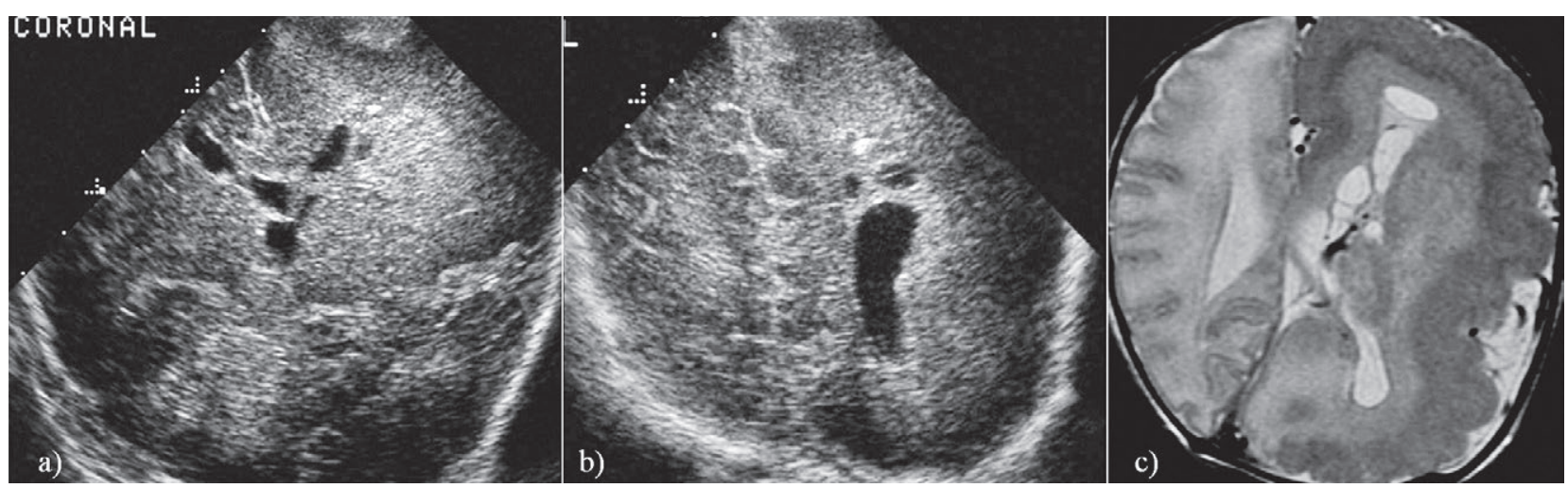

Fig 16. Hemimegalencephaly. Coronal ultrasound images (a, b) show enlarged left cerebral hemisphere with abnormally thick and broad cortices and irregular dilated lateral ventricle on the affected side. On T2-weighted axial MR image (c), there is diffusely enlarged dysplastic left cerebral hemisphere with bizarre shaped, dilated left lateral ventricle. The left cerebral hemisphereconsists of dysplastic tissue with malformations of cortical development. Myelination is accelerated in the affected brain, explaining increased white matter echogenicity on ultrasound and low signal intensity on MR T2-weighted image.
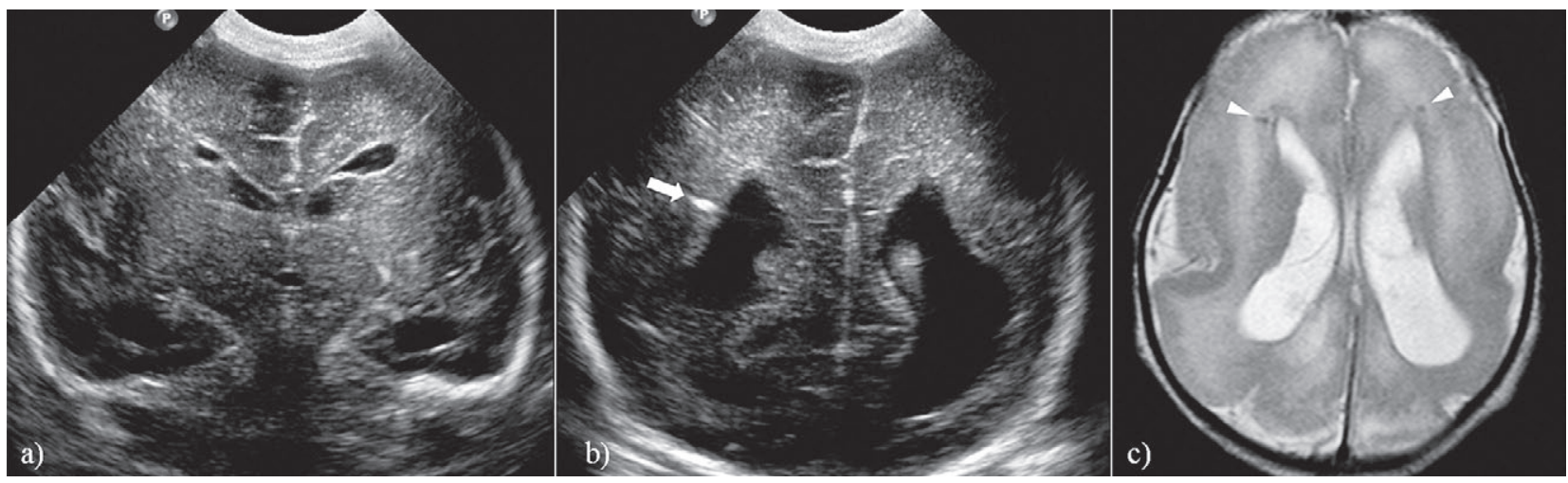

Fig 17. Congenital cytomegalovirus infection. Coronal ultrasound images $(\mathrm{a}, \mathrm{b})$ reveal symmetric lateral ventriculomegaly and bilateral subependymal cysts. Focal periventricular calcification (arrow in b) is noted. When considering this baby's gestational age (38+2 weeks), there is a high suspicion of migration anomaly because there is a lack of sulcation with thick cortices. On MR T2-weighted axial image (c), cortical malformation of pachygyria or polymircogyria is confirmed with bilateral abnormal shallow sylvian fissures. Periventricular calcifications are seen as dark signal dots near the frontal horns of the lateral ventricles (arrowheads in c).

\section{Congenital Cytomegalovirus (CMV) infection}

The most common organism for congenital CNS infection is cytomegalovirus and toxoplasmosis is the second most common cause. The age of the fetus at the time of infection is important: earlier infections affect organogenesis resulting in malformations while later infections cause destructive changes with calcifications [1]. Sonographic findings of congenital cytomegalovirus infections include ventricular dilatation with periventricular calcifications, subependymal cysts, and varying degrees of cortical malformations (fig 17).

\section{Skull defect}

Encephalocele or meningoencephalocele is classified as a neural tube defect with herniation of the brain tissue through a defect in the cranium. Occipital encephaloce- les are most common in the United States and Western countries while frontoethmoidal encephaloceles are more common in Asian. Lateral (or off-midline) cephaloceles can be found along the coronal and lambdoid sutures and they are thought toresultfrom secondary reopening of the neural tube. Ultrasonography may show skull defects through which the brain tissue herniates (fig 18).

\section{Vascular malformations}

Vein of Galen malformation is one of the well-known vascular anomalies occurring in the midline posterior to the third ventricle. It is not a true aneurysmas it was traditionally referred to as, but an arteriovenous fistula [16]. On gray scale images, it appears as an anechoic lesion and the differentiation from the arachnoid cyst can be easily made by a color Doppler study. Dural AV fistula 


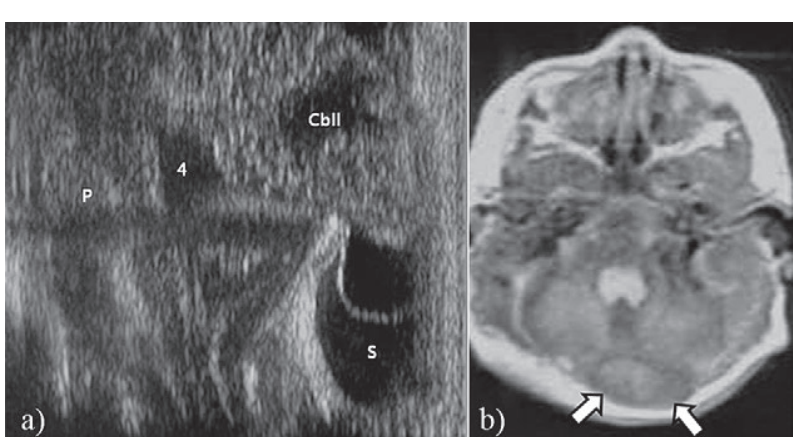

Fig 18. Occipital encephalomeningocele. Close-up ultrasound from the suboccipital approach shows herniated cerebellar tissue and fluid-filled sac (S) through the occipital bone defect. Cbll: cerebellum, P: pons, 4: fourth ventricle. T1-weighted axial MR image (b) confirms occipital meningoencephalocele (arrows).



Fig 19. Dural arteriovenous fistula. Gray-scale ultrasound (a) shows a large round cystic mass (M) in the left posterior aspect compressing the brain parenchyma. Dural arteriovenous fistula can be easily diagnosed with the aid of color Doppler study (b). In this baby, there is also extensive periventricular leukomalacia in the both cerebral hemispheres.

or other vascular malformations not in the midline can be identified on ultrasonography in cases where the lesions are large enough (fig 19).

\section{Conclusions}

With the aid of high-end ultrasound scanner, more or more structural abnormalities can be depicted on screening head ultrasound. Knowledge of normal sonographic findings of ventricles, midline structures, sylvian fissures, and cortices can help us suspect congenital malformations in the neonatal brain before performing MR study.

\section{Conflict of interest: none}

\section{References}

1. Siegel MJ. Brain. In: Siegel MJ. (ed). Pediatric sonography. Philadelphia, PA, Lippincott Williams \& Wilkins, Wolters Kluwer 2011; 43-117.

2. Di Salvo DN. A new view of the neonatal brain: clinical utility of supplemental neurologic US imaging windows. Radiographics 2001; 21: 943-955.

3. Enriquez G, Correa F, Lucaya J, Piqueras J, Aso C, Ortega A. Potential pitfalls in cranial sonography. Pediatr Radiol 2003; 33: 110-117.

4. Lowe LH, Bailey Z. State-of-the-art cranial sonography: Part 2, pitfalls and variants. AJR Am J Roentgenol 2011; 196: 1034-1039.

5. Batton DG, Swails TL. Isolated lateral ventricular asymmetry in very low-birth-weight infants: a left-sided lesion? Am J Perinatol 1998; 15: 183-186.

6. Tubbs RS, Oakes P, Maran IS, Salib C, Loukas M. The foramen of Monro: a review of its anatomy, history, pathology, and surgery. Childs Nerv Syst 2014; 30: 1645-1649.

7. Spalice A, Parisi P, Nicita F, Pizzardi G, Del Balzo F, Iannetti P. Neuronal migration disorders: clinical, neuroradiologic and genetics aspects. Acta Paediatr 2009; 98: 421-433.

8. Supprian T, Sian J, Heils A, Hofmann E, Warmuth-Metz $\mathrm{M}$, Solymosi L. Isolated absence of the septum pellucidum. Neuroradiology 1999; 41: 563-566.

9. Leventer RJ, Jansen A, Pilz DT, et al. Clinical and imaging heterogeneity of polymicrogyria: a study of 328 patients. Brain 2010; 133: 1415-1427.

10. Heard AJ, Urato AC. The isolated mildly enlarged cisterna magna in the third trimester: much ado about nothing? J Ultrasound Med 2011; 30: 591-593.

11. Kollias SS, Ball WS Jr, Prenger EC. Cystic malformations of the posterior fossa: differential diagnosis clarified through embryologic analysis. Radiographics 1993; 13: 1211-1231.

12. Barkovich AJ, Raybaud C. Congenital malformations of the brain and skull. In: Barkovich AJ, Raybaud C. (eds). Pediatric neuroimaging. Philadelphia, PA, Lippincott Williams \& Wilkins, Wolters Kluwer 2012; 367-568.

13. Meijler G. Neonatal cranial ultrasonography. Berlin, Heidelberg: Springer-Verlag, 2012; 97-110.

14. Barkovich AJ, Kuzniecky RI, Jackson GD, Guerrini R, Dobyns WB. A developmental and genetic classification for malformations of cortical development. Neurology 2005; 65: 1873-1887.

15. Abdel Razek AA, Kandell AY, Elsorogy LG, Elmongy A, Basett AA. Disorders of cortical formation: MR imaging features. AJNR Am J Neuroradiol 2009; 30: 4-11.

16. Abbitt PL, Hurst RW, Ferguson RD, Mcllhenny J, Alford BA. The role of ultrasound in the management of vein of Galen aneurysm in infancy. Neuroradiology 1990; 32: 86-89. 